\title{
Trump 2016: ¿presidente gracias a las redes sociales?
}

Roberto Rodríguez-Andrés ${ }^{1}$

Recibido: 2017-08-14

Enviado a pares: 2017-09-04

DOI: $10.5294 /$ pacla.2018.21.3.8
Aprobado por pares: 2017-10-04

Aceptado: 2018-10-01

\section{Para citar este artículo / to reference this article / para citar este artigo}

Rodríguez-Andrés, R. (2018). Trump 2016: ¿presidente gracias a las redes sociales?

Palabra Clave, 21(3), 831-859. DOI: 10.5294/pacla.2018.21.3.8

\section{Resumen}

El manejo de internet y las redes sociales, especialmente Twitter y Facebook, ha sido señalado como uno de los factores que contribuyó al triunfo de Donald Trump en las elecciones de 2016 en los Estados Unidos. En este artículo, se ofrece una descripción de cómo utilizó estas redes el candidato republicano y cuáles fueron sus estrategias digitales, según el seguimiento directo de la campaña y la revisión de informaciones periodísticas, así como el análisis de las investigaciones académicas realizadas hasta el momento. De esta revisión puede concluirse que las redes sociales desempeñaron un papel destacado en estas elecciones, ampliando su poder de influencia con respecto a los anteriores comicios de 2008 y 2012, en los que Obama ya hizo un uso extensivo de ellas, y que Trump aventajó a Hillary Clinton a la hora de utilizarlas para movilizar a sus públicos prioritarios, desmotivar a los de la candidata rival y lanzar sus mensajes, lo cual constituyó una parte importante de su victoria. No obstante, se concluye también que no podría afirmarse que las redes fueron, por sí solas, el motivo principal del éxito del republicano, ya que en su triunfo han de encontrarse

1 orcid.org/0000-0003-4262-8237. Universidad Pontificia Comilla, España.rrodrigueza@comillas.edu 
otras razones de fondo vinculadas con el contexto político-social en el que se desarrollaron estas elecciones.

\section{Palabras clave}

Redes sociales; internet; Twitter; Facebook; Cambridge Analytica; Trump; comunicación política (Fuente: Tesauro de la Unesco). 


\section{Trump 2016: ¿President Elected Thanks to Social Media?}

\section{Abstract}

The Internet and social media, particularly Twitter and Facebook, have been identified as one of the factors that contributed to Donald Trump's triumph in the United States presidential elections of 2016. In this paper, we describe how the Republican candidate used social media and what his digital strategies were, after directly monitoring the campaign and reviewing journalistic information, as well as the analysis of the academic research carried out so far. Based on this review, it is possible to conclude that social media played a significant role in these elections, expanding its power of influence as compared to the previous elections of 2008 and 2012, when Obama used them extensively, and which Trump used better than Hillary Clinton to move his priority audience, to discourage his rival's audience, and to send his messages, thus making it an important part of his victory. It is also concluded, however, that it is wrong to say that social themselves were the main reason for the Republican candidate's success, as there are other substantial reasons to his triumph which are linked to the socio-political context in which these elections took place.

\section{Keywords}

Social media; Internet; Twitter; Facebook; Trump; political communication (Source: Unesco Thesaurus). 


\section{Trump 2016: presidente eleito graças às redes sociais?}

\section{Resumo}

O uso da internet e das redes sociais, especialmente o Twitter e o Facebook, foi indicado como um dos fatores que contribuiu para o triunfo de Donald Trump nas eleições presidenciais de 2016 nos Estados Unidos. Neste artigo, apresenta-se uma descrição de como o candidato republicano utilizou essas redes e quais foram suas estratégias digitais, segundo o acompanhamento direto da campanha e a revisão de informações jornalísticas, bem como a análise das pesquisas acadêmicas realizadas até o momento. Com base nessa revisão, pode-se concluir que as redes sociais desempenharam um papel de destaque em tais eleições, ampliando seu poder de influência com respeito às eleições anteriores de 2008 e 2012, quando Obama já havia feito um uso extensivo delas e Trump se beneficiou mais do que Hillary Clinton na hora de utilizar as redes para mobilizar seu público prioritário, desmotivar a audiência da candidata rival e lançar suas mensagens, o que constituiu uma parte importante da sua vitória. No entanto, conclui-se também que não é possível afirmar que as redes foram, por si só, o motivo principal da vitória do republicano, já que é possível encontrar em seu triunfo outras razões de fundo vinculadas ao contexto político-social no qual ocorreram essas eleições.

\section{Palavras-chave}

Cambridge Analytica; comunicação política; Facebook; internet; redes sociais; Trump; Twitter (Fonte: Tesauro da Unesco). 


\section{Introducción}

Tras la inesperada victoria de Donald Trump en las presidenciales estadounidenses de noviembre de 2016, muchos medios de comunicación, analistas y académicos empezaron a preguntarse por qué habían fallado los sondeos y, en último término, cuáles habían sido las razones del éxito del candidato republicano. Y, entre ellas, hubo amplia coincidencia en torno al papel que habrían podido tener las redes sociales, especialmente Facebook y Twitter, en esta victoria (Cellan-Jones, 2016; Groshek y Koc-Michalska, 2017; Breur, 2017).

El propio candidato, en una entrevista en el programa 60 minutos (CBS) emitida el 13 de noviembre de 2016, atribuyó una parte importante de su triunfo al uso que había hecho de las redes. "El hecho de que tenga ese poder en términos de números con Facebook, Twitter, Instagram, etcétera, creo que me ayudó a ganar en una carrera en la que otros gastaron más dinero que yo" (Morin, 2016).

En tal contexto, esta investigación se plantea como objetivo profundizar en cómo fue la estrategia y el uso de las redes sociales por parte del equipo de campaña de Trump, así como tratar de comprobar si, como muchos apuntaron, estas fueron decisivas para llevarlo hasta la Casa Blanca. Para ello, este artículo se plantea desde el punto de vista metodológico como una revisión basada en tres ejes fundamentales: 1) la observación empírica de la campaña y el seguimiento de las redes sociales de Trump por parte del autor, 2) un exhaustivo seguimiento de informaciones periodísticas (publicadas, principalmente, en los Estados Unidos) durante 2016 y 3 ) la revisión de los primeros trabajos e investigaciones académicas que se han publicado en torno a estos comicios y el papel que en ellos desempeñaron las redes sociales.

\section{El equipo digital de Trump}

Donald Trump no tuvo un equipo digital tan amplio como el de Hillary Clinton ni tampoco el asesoramiento de grandes gurús en el ámbito de la comunicación política 2.0, como fue el caso de la candidata demócrata (Rodríguez, 2016). A pesar de ello, a su favor jugaba el hecho de tener ya una 
presencia consolidada en las redes sociales, acrecentada durante su época como presentador de un famoso reality televisivo.

El director digital de su campaña fue Brad Parscale, diseñador web que trabajaba desde 2011 haciendo páginas web para empresas de Trump, pero que no contaba con ninguna experiencia en política. Este hecho, unido a la reducida dimensión de su compañía, hizo que se cuestionara su capacidad para dirigir una campaña presidencial, sobre todo cuando se produjeron errores como la caída de la web del candidato en el primero de los debates con Clinton el 26 de septiembre de 2016 o el fracaso de los primeros correos electrónicos de recaudación de fondos, que en más de un $60 \%$ fueron bloqueados como spam (Marshall, 2016; Winston, 2016). Sin embargo, al final, la victoria de Trump hizo que Parscale pasara de ser un personaje casi desconocido a tener reconocimiento mundial, señalándolo abiertamente como uno de los principales responsables del triunfo (Abdullin, 2016; De la Calle, 2016; Lapowsky, 2016; Petovel, 2016; Zanoni, 2016).

Parscale fue uno de los primeros en incorporarse al equipo de campaña de Trump, en junio de 2015, cuando empezaba la carrera por las primarias (Thomas, 2015). Como él mismo ha descrito, diseñó la web del candidato en solitario, desde la sala de estar de su casa en San Antonio, y esbozó un primer borrador de estrategia en redes sociales (Miller, 2016). Poco a poco, fue creando su propio equipo, que al final de la campaña estaba formado por más de un centenar de personas (Lambrecht, 2016) y progresivamente fue ganando también peso en el equipo electoral de Trump, hasta convertirse en uno de sus principales asesores (Miller, 2016). De esta progresión dice mucho cómo fueron aumentando sus ingresos, que pasaron de los USD 1500 que cobró por hacer la primera web para la nominación a los USD 90 millones que percibió en total por su trabajo en la campaña (Locklear, 2016).

\section{La apuesta por las redes sociales}

Uno de los elementos más subrayados de la estrategia de campaña de Trump, y más innovadores con respecto a campañas anteriores, es que confió en las redes sociales como primer canal de comunicación con el electorado, dándoles más importancia que a los medios tradicionales (Hendricks, 2017). 
Por ejemplo, desde los inicios de campaña, su director digital hizo ver a Donald Trump que no merecía la pena malgastar dinero en anuncios en televisión, medio en el que el candidato ya tenía gran repercusión gracias a sus explosivas declaraciones. Así se comprobó en un estudio llevado a cabo por The New York Times y la firma SMG Delta en febrero de 2016, en plenas primarias en ambos partidos (Confessore \& Yourish, 2016). Mientras que candidatos republicanos como Bush o Rubio habían gastado en publicidad televisiva USD 82 y USD 55 millones, respectivamente, la inversión de Trump era tan solo de USD 10 (también por debajo de los USD 27.9 de Clinton). Sin embargo, según este mismo estudio, y tal como se muestra en la figura 1, si hubiera tenido que pagar por las noticias que consiguió gratuitamente en las televisiones, habría tenido que gastar USD 1898 millones, cifra considerablemente más alta que la del resto de candidatos. Y a esta conclusión llegaron también algunas investigaciones académicas (Wells et al., 2016), en las que se constató la pericia del candidato republicano para ejecutar una "campaña híbrida" donde el entorno online acabó impactando decisivamente en los medios tradicionales.

Figura 1. Inversión publicitaria en televisión vs. espacio ganado gratuitamente en este medio

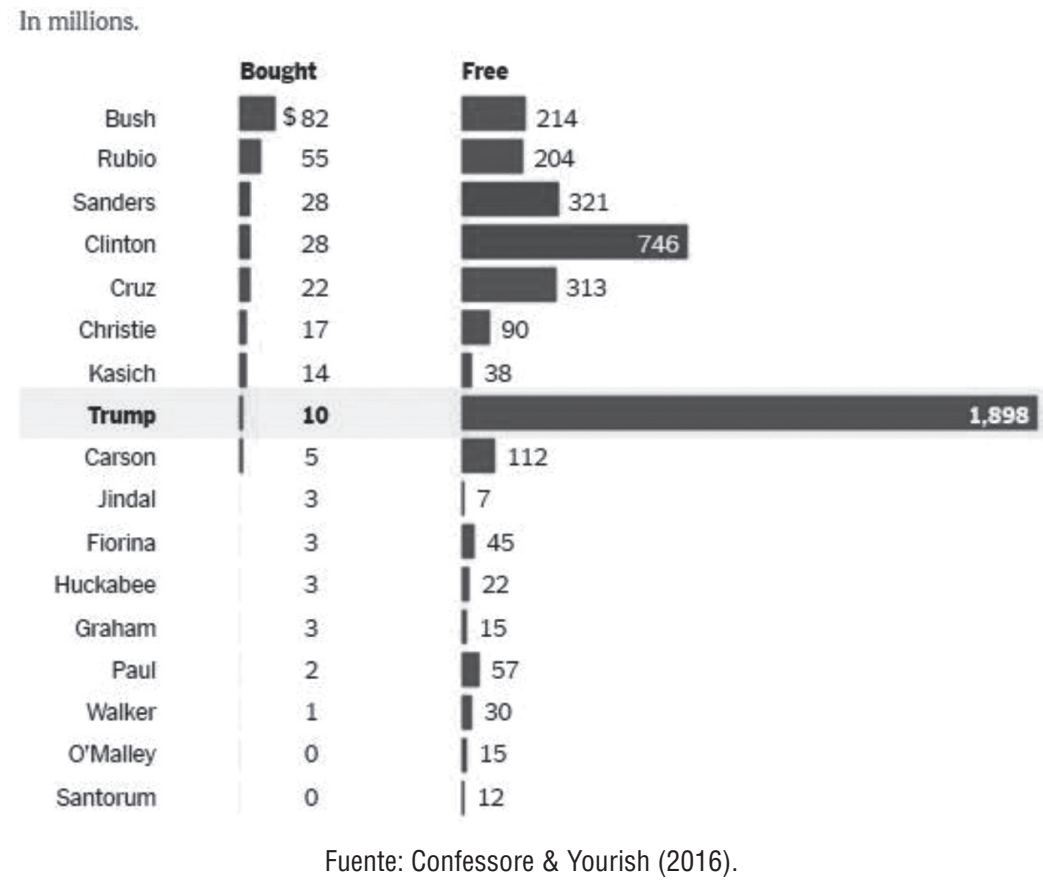


Conscientes de esta repercusión, el equipo de campaña consideró que era mejor centrar los esfuerzos económicos en el entorno digital. Así, como describe Petovel (2016), "gran parte de la inversión de televisión y otros medios derivó a Facebook, Google, Twitter, YouTube y otras redes sociales más nuevas como Snapchat, Instagram, Vine o Periscope”. El director digital de Trump reconoció que "esto nos permitió competir contra la maquinaria de Hillary con una inversión mucho más efectiva” (Lambrecht, 2016). Y no dudó en subrayar que las redes sociales fueron claves para el éxito: "Creo que esas plataformas nos ayudaron a ganar la campaña” (Goins-Phillips, 2016).

Si se observa la dimensión meramente cuantitativa, esta estrategia tuvo sus frutos, ya que Trump consiguió sobrepasar a Clinton. Según datos recogidos para esta investigación, el último día de campaña lideró en número de visitas a sus páginas web ( 3.5 millones mensuales frente a 1.2 de Clinton) y también en seguidores en las principales redes sociales. En Twitter, Trump consiguió 13.9 millones frente a 10.6 de Clinton, cuando, como narran Davis, Holtz-Bacha y Just (2016), un año antes del día de la votación prácticamente tenían los mismos seguidores (4.7 millones Trump frente a 4.6 Clinton). Esta superioridad se vio reflejada también en Facebook ( 13.3 frente a 8.8 millones) e Instagram (4 frente a 3.7). La única de las grandes redes en la que Clinton superó al candidato republicano fue YouTube (103000 suscriptores en el canal de Trump frente a los 135000 de Hillary). Pero no solo fue una cuestión de superioridad numérica, puesto que diversos estudios mostraron también que el engagement de los seguidores de Trump casi doblaba al de sus competidores en las primarias (Meyer, 2016; Pérez-Martínez, Rodríguez-González y Tobajas-Gracia, 2017) y que, ya en la campaña, estaban también mucho más polarizados y movilizados que los de Clinton (Bovet, Morone y Makse, 2016; Wang, Yuncheng y Jiebo, 2016; Malec, Pavlicek y Luc, 2016).

La relevancia de Trump se evidenció, asimismo, en el número de búsquedas en Google (Fraile-Garrido, 2016) y en la cantidad de menciones que recibió durante la campaña en las redes sociales (Smith, 2017; Francia, 2017). Un análisis llevado a cabo por la consultora Social Flow en marzo de 2016 reveló que, si una persona tuviera que leer todo lo que se había escrito de Donald Trump en las redes hasta ese momento, tardaría más de 1200 años en hacerlo (frente a los 874 de Sanders y Clinton juntos) (Dailey, 2016). 
Esta superioridad en menciones fue especialmente clara en el caso de Twitter. Tal como se aprecia en la figura 2, desde el 1 de enero de 2016 y hasta el día de la elección, Trump concitó mayor número de tuits durante todo el periodo, incluso en los tres debates presidenciales, con la única excepción de la Convención Demócrata y los últimos días de campaña (Coyne, 2016).

\section{Figura 2. Evolución del número de menciones en Twitter a Trump y Clinton del 1 de enero al 7 de noviembre de 2016}

\section{The Twitter Election}

Tweets mentioning each candidate this year

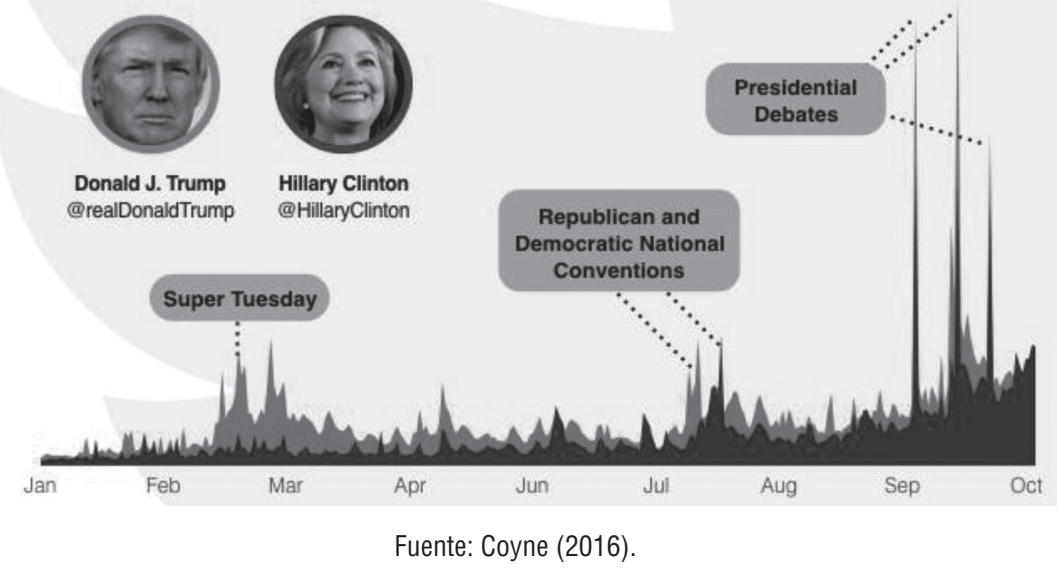

Algunos análisis académicos han apuntado que esta superioridad pudo haberse fomentado de forma artificial por el equipo de Trump al crear informáticamente miles de bots o cuentas ficticias desde las que amplificar sus mensajes en Twitter, dominar la conversación y generar trending topics, práctica que también habría desarrollado el equipo demócrata, aunque con menor intensidad. Así se comprobó en un estudio de la Universidad del Sur de California (Bessi y Ferrara, 2016) que analizó 20 millones de tuits generados entre el 16 de septiembre y el 21 de octubre y escritos por unos 2.8 millones de usuarios. De ellos, se comprobó que aproximadamente 400000 eran bots, los cuales generaron el $19 \%$ de la conversación total, distorsionando claramente el debate en Twitter, en su gran mayoría a favor de Trump. Resultados similares fueron obtenidos por investigadores de la Universidad de Oxford, dirigidos 
por Philip Howard, que hicieron seguimiento a más de 19 millones de tuits entre el 1 y el 9 de noviembre (Kollanyi, Howard y Woolley, 2016) y también por Hermida (2016), que llegó a contabilizar que el 80 \% de la actividad en Twitter a favor de Trump fue generada por estos bots.

\section{La apuesta por el big data}

Además de apostar por las redes sociales, Parscale también convenció a Trump de la importancia de invertir en datos, a pesar del escepticismo inicial que había mostrado públicamente el candidato al considerar que se estaba "sobrevalorando" la importancia de los datos en las campañas (Bykowicz, 2016; Delany, 2016; Vogel y Samuelshon, 2016). Superada esta reticencia inicial, empezaron a trabajar con encuestas online, llamadas telefónicas y big data para conocer mejor a los electores, utilizando esta información para recaudar fondos y centrar mensajes y publicidad en los medios prioritarios y en los lugares y electores clave (sobre todo entre los blancos descontentos con la política tradicional) (Abdullin, 2016; De la Calle, 2016; Lapowsky, 2016; Conley, 2017). Según Parscale, "usamos los datos como nadie antes había hecho en la historia del Partido Republicano" (Spriester, 2016).

Todo este trabajo de datos se sustanció en la puesta en marcha de una gran base, bajo el nombre de Proyecto Álamo, que logró reunir información de 14 millones de votantes "indecisos pero persuadibles" en los estados decisivos (Green y Issenberg, 2016). Este trabajo se complementó con la base de datos del Comité Nacional Republicano y se reforzó, asimismo, con el asesoramiento de la compañía británica Cambridge Analytica. Esta firma, especialista en realizar perfiles psicológicos de votantes, había trabajado para la campaña del brexit y para el senador Ted Cruz durante las primarias, y disponía de una base de datos de más de 220 millones de adultos estadounidenses (Hope, 2016; Johnson, 2016; Vogel y Samuelshon, 2016; Winston, 2016; González, 2017). Los datos, según el director digital de campaña, fueron cruciales para Trump: "Por eso ganamos. Conocíamos perfectamente a los votantes que necesitábamos convencer y los convencimos a gran escala” (Lambrecht, 2016). 
Pero, además de atraer a estos electores, el equipo digital centró también sus esfuerzos en minar la imagen de Hillary Clinton entre sus propios votantes potenciales, aquellos sin cuyo apoyo tendría más difícil la victoria, especialmente "blancos liberales idealistas", mujeres jóvenes y afroamericanos. Propiciaron la denominada voter suppression (supresión de votos) entre votantes de Clinton, sembrando en ellos la duda acerca de su propia candidata, invitándolos para que se quedaran en casa y no la votaran. Para ello, desplegaron una intensa campaña de anuncios negativos en las redes sociales subrayando los aspectos más discutidos de su trayectoria (Abdullin, 2016; Bump, 2016; Green y Issenberg, 2016; McCabe, 2016; Miller, 2016). Según Winston (2016), los 150 millones de dólares que el equipo de Trump invirtió en anuncios en Facebook e Instagram durante las últimas semanas de campaña dirigidos a estos colectivos constituyeron "la operación de voter suppression más exitosa de toda la historia de los Estados Unidos”.

Quizá el anuncio más destacado dentro de esta estrategia fue el Superpredators. Utilizando una animación al estilo de la serie South Park, este spot acusaba a Clinton de racista por un discurso que pronunció en 1996, cuando era primera dama, en el que, hablando de los crímenes violentos cometidos por jóvenes negros en distintos puntos del país, afirmó que estos jóvenes se comportaban como unos "superdepredadores" (Byrnes, 2016). Según el equipo de campaña de Trump, ese vídeo consiguió dejar en casa a un buen número de electores negros que en circunstancias normales habrían votado por Hillary (Winston, 2016).

\section{La campaña en Twitter}

En la campaña digital de Trump, hubo dos redes sociales con especial protagonismo: Twitter y Facebook. En el primer caso, solo en los Estados Unidos se escribieron un billón de tuits sobre las elecciones desde agosto de 2015 (cuando empezaron los primeros debates de las primarias) hasta el día de la votación (Coyne, 2016). Y el segundo debate entre Trump y Hillary fue el más tuiteado de la historia, con más de 17 millones de mensajes (Rosenbaum, 2016). Para algunos, esta campaña ha sido la primera que puede recibir la denominación de "Twitter election” (Heller, 2016). Pero, además de esta dimensión cuantitativa, algunas investigaciones han 
subrayado también que durante estos comicios los mensajes de los candidatos en Twitter pudieron ser más influyentes ante los votantes que las noticias difundidas por los medios tradicionales, lo cual refuerza el papel de esta red social (Morris, 2017).

En el protagonismo de Twitter, desempeñó un papel muy destacado el propio Trump, que la convirtió en una de sus principales herramientas de comunicación. Según Michael Barbaro (2016), periodista de The New York Times, “Trump ha dominado Twitter de una forma que ningún candidato a presidente ha conseguido hasta ahora. Liberó y redefinió su poder como herramienta para promoción política, distracción y ataque”.

Ya se ha mencionado la superioridad del republicano en número de seguidores y de menciones en esta red social durante la campaña. Del análisis de su cuenta de Twitter, junto con la opinión de expertos en comunicación política 2.0 y diversas investigaciones académicas, se pueden sintetizar algunos factores que pudieron contribuir a este éxito.

El primero de ellos fue la implicación personal del candidato en esta red social. El propio Trump fue quien escribió la mayoría de los mensajes, algo que suele ser del agrado de la comunidad tuitera, en contra de lo que ocurría con la cuenta de Clinton, mayoritariamente mantenida por su equipo. El candidato republicano tuiteaba constantemente, incluso a altas horas de la madrugada, en lo que pronto empezó a denominarse como las tweet storms (tormentas de tuits) de Trump (Gallagher, 2017). Como afirmó Mike Berland, consejero de Edelman Berland, asistimos a "un continuo acto de campaña en Twitter a todas horas" (Alaimo, 2016). Y de ello da buena muestra la comparación del número de tuits escritos en campaña por Trump (34 000) y Clinton (9800). El republicano escribió más, pero también compartió e interaccionó con sus seguidores en mayor medida que su rival, tal como comprobó un grupo de investigadores de las universidades de Lehigh y Ámsterdam (Lee y Lim, 2016), que constataron que, mientras que las tres cuartas partes de los mensajes de Clinton fueron tuits o mensajes propios, más de la mitad de los de Trump eran retuits y respuestas a los ciudadanos. 
El segundo factor, vinculado estrechamente con el primero, fue la naturalidad y espontaneidad que se desprendía de la cuenta en Twitter de Donald Trump. Para los tuiteros, resultaba muy fácil identificarlo detrás de cada uno de sus mensajes, para lo bueno y para lo malo. Su imagen en Twitter no difería de la que estaban acostumbrados a ver en televisión. Según Enli (2017), podría decirse que la estrategia de campaña de Clinton en redes sociales fue más profesionalizada, mientras que la de Trump fue más auténtica y con un estilo casi amateur, "poco ortodoxa", en palabras de Berger (2017), lo que contrariamente a lo que podría parecer fue su principal ventaja. De la misma opinión es Tinaquero (2016), para quien "Trump fue auténtico y dio a su audiencia lo que quería".

El tercer factor fue precisamente lo que dio a su audiencia a través de Twitter, sobre todo a los ciudadanos estadounidenses enfadados con la política tradicional. Mientras que la cuenta de Clinton, salvo contadas excepciones, estuvo regida por la moderación y lo políticamente correcto, Trump incumplió todas las convenciones establecidas en comunicación política acerca del uso que se debe hacer de esta herramienta (Rodríguez y Ureña, 2011). Fue rompedor, irreverente, provocador, insultante, "agresivo y ofensivo" (Plantic, Ratkic y Suput, 2017, p. 379). En cierta medida, como ha subrayado Ott, esta campaña sirvió para demostrar que Twitter "privilegió un tipo de discurso simple, impulsivo y también incívico” (2017, p. 59). Pero para muchos, sobre todo para esa mayoría silenciosa que le dio el triunfo, Trump fue el único que hablaba claro, diciendo lo que muchos pensaban y nadie se atrevía a decir. Y además, usando recursos muy bien valorados en esta red social, como la sátira, la ridiculización y el entretenimiento, por ejemplo con un uso extensivo de memes como herramienta política (Milner y Phillips, 2016). En definitiva, y en contra de lo que hacía Clinton, Trump no buscaba contentar a todos, sabía bien a quién dirigirse y cómo movilizar a través de Twitter. Como explicó el consultor político Carlos Gutiérrez (2016), "a través de un discurso agresivo, dirigido a la población nativa del país y apelando al sentimiento patrio, buscaba ganar los votantes necesarios que lo llevasen a la presidencia [...]. Su posición le restó apoyos entre cierta población, pero dentro de su población objetivo, le sumó más simpatías". De hecho, según un análisis efectuado por investigadores de la Universidad de Rochester, cuanto más irreve- 
rente se mostraba, más crecía su número de seguidores y más apoyo recibía de ellos (Wang et al., 2016).

En sentido inverso, se podría afirmar que, cuanto más políticamente incorrecto era, más aumentaban las críticas que recibía, pues fue el candidato más criticado en redes sociales durante toda la campaña por el resto de postulantes (Gross y Johnson, 2016; Benoit y Glantz, 2017). Pero eso no hizo más que aumentar su repercusión, lo cual, en el fondo, acabó siendo favorable para su campaña y constituye el cuarto factor de éxito de su utilización de Twitter. Trump intentaba demostrar continuamente que el establishment estaba contra él. Cuanto más lo atacaban, más legitimidad daban a su discurso victimista, con lo que involuntariamente acabaron entrando en su juego (Tinaquero, 2016). Como describió gráficamente el decano de la Escuela de Periodismo de la Universidad de Columbia, Steve Coll, en una entrevista al diario El País, “Trump usó esto como carburante para su campaña. Les dijo a sus seguidores, 'veis, van por nosotros, pero yo hago campaña por vosotros"” (Aguilar, 2016). A pesar de ello, se ha difundido que en algún momento su propio equipo le habría recomendado rebajar el tono de sus mensajes. Fue el caso del periódico The New York Times, según el cual, en los últimos días de campaña, con los sondeos muy ajustados, le prohibieron tuitear sin supervisión previa, por miedo a que una nueva polémica pudiera afectar el resultado (Haberman et al., 2016). De hecho, en la última semana de campaña, resultó especialmente revelador que Trump publicara menos mensajes en Twitter que Clinton (103 frente a 316), cuando su tónica de toda la campaña había sido la contraria (Davis, 2017).

El quinto de los elementos que contribuyó a su éxito en Twitter es que identificó en todo momento un enemigo claro, que coincidía con las fobias de sus seguidores (Estado Islámico, globalización, establishment, inmigrantes irregulares, medios de comunicación tradicionales, etc.). Y lo mismo hizo con sus rivales, a quienes ponía en la diana de sus ataques, entrando en el cuerpo a cuerpo en las redes, casi a modo de "trol”, como definió Joe Rospars, jefe de estrategia digital de Obama en 2008 y 2012 (Hall, 2016). En palabras del consultor Gutiérrez-Rubí (2016), "el republicano ha reducido la política a un juego de rol: con sus villanos y sus escenarios. Trump no explica problemas, 
identifica culpables $[\ldots]$. Y encontrar culpables (y etiquetarlos) es un poderoso factor de movilización". Algunas investigaciones han probado que los tuits en los que se mostraba más duro con sus rivales eran los que acumulaban más apoyo (Wang et al., 2016; Kirk y Martin, 2017).

Por último, el sexto factor es que consiguió influir de forma muy destacada en la agenda mediática gracias a Twitter. Trump usó esta red social para dirigirse directamente a sus votantes, saltando el filtro de unos medios que, en su gran mayoría, eran abiertamente hostiles hacia él. Pero Twitter fue también el altavoz desde el que lanzó sus mensajes para que fueran recogidos por esos medios. Así lo resumía Mindy Finn, asesora de Bush y Romney: "Ve a Twitter primero, siembra la controversia y deja que los medios de comunicación hablen de ti en las próximas 24 horas" (Hall, 2016).

\section{La campaña en Facebook}

Según Zac Moffatt, director digital de Romney en 2012, "todo el mundo pensó que 2008 fue la elección de Facebook, pero yo diría que 2016 ha sido la elección de Facebook" (Mims, 2016). Según este consultor, el valor actual de esta red social es que permite llegar a tres de cada cuatro estadounidenses en una sola plataforma y de una forma muy efectiva.

La campaña de Trump, consciente de este poder, utilizó Facebook con tres objetivos principales: recaudar fondos a través de pequeñas donaciones (alcanzando los USD 250 millones por esta vía), difundir mensajes a públicos prioritarios a través del microtargeting que permite esta red social (como los destinados a la voter suppression de votantes de Clinton) y diseminar noticias.

Sobre este último punto, tuvo un papel muy relevante la contratación de darkposts o publicaciones invisibles (Green y Issenberg, 2016), así como la utilización de Facebook Live para emitir vídeos en directo. Como ha analizado Gutiérrez-Rubí (2016), aunque Trump empezó a usar esta plataforma tan solo un mes antes de los comicios (mucho después que Hillary), al final acabó sobrepasándola también en número de retransmisiones ( 47 más que la demócrata). Además, por cada una de ellas, Trump logró 150000 comentarios, mientras que Clinton solo 69000. 
Pero, sin duda, el aspecto más relevante ( $y$ también el más controvertido) de la campaña en Facebook fue la utilización de esta red para la difusión de noticias falsas, asunto en el que aún se investiga la posible implicación del Gobierno ruso a favor de Trump y que ha llevado, incluso, a los propietarios de Facebook a pedir disculpas y a adoptar medidas para evitar que esto pueda volver a repetirse.

En esta campaña, se crearon cientos de páginas web fantasma desde las que se lanzaron noticias falsas a favor del candidato o en contra de Clinton, las cuales lograron que estas fueran ampliamente difundidas por Facebook (Winston, 2016). Noticias como el apoyo del papa Francisco a Donald Trump, el abuso sexual por parte de Bill Clinton a una niña de 13 años, que Hillary Clinton había vendido armas al Estado Islámico, que había confesado en un correo electrónico que ella creó este grupo terrorista, o que había mandado asesinar a un agente del FBI que la investigaba (Benton, 2016). Por muy disparatadas que pudieran parecer, estas noticias tuvieron amplio eco y sirvieron, sobre todo, para reforzar a los propios votantes de Trump. Según un estudio de Buzzfeed realizado en los últimos tres meses de campaña, estas noticias falsas lograron tener más impacto que las de los principales medios de comunicación: mientras que las primeras acumularon nueve millones de reacciones y comentarios en Facebook, las segundas solo tuvieron siete millones (Silverman, 2016). Otro estudio de Ipsos mostró también que el $75 \%$ de los estadounidenses expuestos a estas noticias falsas en Facebook las dio como verdaderas (Silverman y Singer-Vine, 2016). Por último, una investigación de Allcott y Gentzkow (2017) para The National Bureu of Economic Research de los Estados Unidos, constató que, de entre todas las noticias falsas que circularon en los últimos tres meses de campaña en Facebook, las que beneficiaban a Trump fueron compartidas treinta millones de veces, mientras que las favorables a Clinton solo llegaron a los ocho millones.

En definitiva, se construyó una realidad alternativa, que se encuadra en lo que ha venido denominándose en los últimos años como el triunfo de la posverdad (post-truth), concepto con el que se alude a aquellas mentiras difundidas con apariencia de verdad y lanzadas, además, con una fuerte carga emocional, las cuales acaban siendo más influyentes para la opinión 
pública que la propia verdad (Suiter, 2016). Estas noticias encuentran amplia difusión en las redes sociales, lo que les permite llegar a públicos cada vez más amplios, al haber suplantado a los propios medios de comunicación tradicionales como fuente principal de información. De hecho, según el Pew Research Center, el 62 \% de los estadounidenses confiesan que consultan habitualmente noticias a través de las redes, principalmente Facebook y Reddit (Gottfried y Shearer, 2016) y el $44 \%$ se informaron de la campaña a través de ellas (Williams, 2017).

\section{Conclusiones: ¿ganó Trump por las redes sociales?}

Las redes sociales desempeñaron un destacado papel en las elecciones estadounidenses de 2016, aumentando su poder con respecto al uso que ya se hizo de ellas en 2008 y 2012. Y a la vista de lo expuesto en este artículo, se puede concluir que el equipo de Trump supo sacar el máximo provecho de estas plataformas de comunicación para movilizar a sus propios electores, desmovilizar a parte de los votantes de Clinton y ganar protagonismo mediático. Esto es lo que ha llevado a analistas e investigadores a considerar que las redes sociales fueron determinantes para que Trump lograra la victoria. Según Alaimo (2016), profesora de la Universidad Hofstra y exmiembro de la Administración Obama, "el uso estratégico que hizo de las redes sociales lo impulsó hacia la presidencia [... . Aunque Trump parezca la antítesis de Obama, los dos [... son sorprendentemente parecidos en dos aspectos: ambos eran candidatos antiestablishment que ganaron la presidencia al superar a sus oponentes en las redes sociales".

Esta opinión es compartida por consultores y expertos en redes. El cofundador de Social Flow, Frank Speiser, señaló que "estas han sido las primeras elecciones en las que han influido realmente las redes sociales" (Lang, 2016). También el CEO de Salesforce, Marc Benioff, uno de los líderes más influyentes de Silicon Valley, manifestó que Trump ganó gracias a las redes, especialmente Twitter: "Creo que Twitter es más importante que nunca [...]. Sin Twitter, no creo que tuviéramos hoy como presidente electo a Trump" (Wagner, 2016). Para Petovel (2016), "la mercadotecnia de redes 
y el email marketing fueron claves en la victoria de Donald Trump". Y Tinaquero (2016) ha descrito gráficamente que, si Roosevelt fue el presidente de la radio, Kennedy el de la televisión y Obama el de internet, Trump es el de las redes sociales.

A tenor de estas reflexiones, cabría preguntarse si se ha producido un cambio sustancial en la comunicación política contemporánea y si las redes sociales han consolidado ya su poder como principal plataforma para la difusión de mensajes, la relación con los ciudadanos y la conformación de la opinión pública, habiendo sustituido a los medios tradicionales. Puede que estemos muy cerca de este momento, pero en este caso concreto creemos que aún es prematuro afirmar que las redes justificaron por sí solas el triunfo de Trump en las elecciones. Porque el éxito de Trump, como ha ocurrido en otros procesos electorales de los últimos años, está íntimamente ligado a su personalidad y, sobre todo, a un momento político muy específico, con grandes niveles de polarización y de desafección ciudadana hacia la política tradicional y esperanzas de cambio. En definitiva, como han apuntado autores como Hendricks (2017), Persily (2017) o Sáez-Mateu (2017), no puede decirse que las redes por sí solas dieran la victoria a Trump, puesto que esta se debió a una compleja intersección de diversas circunstancias tanto políticas como de comunicación, de las que no había habido precedentes en los Estados Unidos. Este contexto fue decisivo para su triunfo y, de no haberse producido, por muy exitosa que hubiera sido su campaña en redes, quizá no hubiera llegado a la Casa Blanca.

En cualquier caso, la experiencia de estas elecciones debe servir a la comunidad científica para seguir investigando sobre el papel de internet y las redes sociales en la comunicación política. Una investigación centrada en tres ejes fundamentales. El primero de ellos versa sobre la capacidad que han alcanzado las redes para convertirse en poderosas herramientas de difusión de información y de comunicación con los ciudadanos, habida cuenta de su amplísima generalización en las sociedades actuales, sobre todo gracias a los teléfonos móviles. Vivimos en una sociedad hiperconectada en la que, según se ha expuesto en este trabajo, cada vez son más los votantes que se informan de las campañas a través de las redes, que han visto así aumentar su poder con 
respecto a los medios tradicionales, algo que seguirá acrecentándose en el futuro. Y la campaña online de Trump ha sido un buen ejemplo de cómo sobreponerse a una prensa, una radio y una televisión mayoritariamente contrarias a sus intereses gracias precisamente a las redes.

El segundo eje, más allá de su poder como difusoras de información, debe centrarse en seguir profundizando sobre la capacidad persuasiva de las redes, es decir, cuál es su influencia en términos de consecución de votos. Admitiendo que en esta campaña ha habido otros factores determinantes para la victoria de Trump, principalmente el hastío hacia la política tradicional, no se puede negar que las redes permitieron al candidato republicano convencer a un buen número de votantes tanto para que le apoyaran a él como para que dejaran de votar a Clinton. Cuántos votos se consiguen gracias a las redes sociales es una pregunta que la comunidad investigadora lleva formulándose en reiteradas ocasiones en los últimos años, sin que aún hayamos encontrado respuestas claras. Es verdad que hemos avanzado en cuestiones clave, constatadas también en esta campaña estadounidense de 2016, como el poder predictor que tienen las redes (principalmente Twitter) como oráculos de los resultados electorales, llegando a cuestionar, incluso, a los propios sondeos, que tan estrepitosamente han fallado en estos comicios. Y también sobre el papel de los algoritmos a la hora de profundizar en la polarización política de los ciudadanos, en un contexto en el que las redes solo muestran mensajes que sintonizan con las preferencias políticas de los usuarios, lo que en el fondo sirve para reforzar aún más las propias convicciones, ocultando impactos de la parte contraria que permitirían escuchar un discurso alternativo que pudiera hacer variar esa opinión.

Por último, el tercer eje está más relacionado con las implicaciones éticas y las consecuencias que las redes sociales pueden tener sobre los procesos electorales y, en último término, sobre las democracias. Mucho se ha escrito sobre las potencialidades de las redes para propiciar una mejor comunicación y relación con los ciudadanos y para fomentar la transparencia, la escucha activa y el diálogo entre políticos y ciudadanos. Pero también urge seguir investigando sobre los peligros que pueden estar comportando, como la ya citada polarización extrema de la opinión pública, la difusión de la posverdad, la 
simplificación y espectacularización del mensaje político o su uso como arma de ataque y de juego sucio contra los rivales, elementos que se han dado cita también en las elecciones de 2016 en los Estados Unidos y en los que Trump ha sido especialmente activo.

\section{Referencias}

Abdullin, R. (2016, noviembre 16). The man who brought victory Trump: The secrets of the cunning campaigns. En Center for Strategic Assessment and Forecasts. Recuperado de https://goo.gl/0q5uhX

Aguilar, A. (2016, noviembre 27). El ambiente está contaminado por noticias falsas. En El País. Recuperado de https://goo.gl/ucGe0t

Alaimo, K. (2016, noviembre 17). Redes sociales: donde Donald Trump tiene su verdadero poder. En CNN Español. Recuperado de https:// goo.gl/bfrlzJ

Allcott, H. \& Gentzkow, M. (2017). Social media and fake news in the 2016 election. En NBER WorkingPaper, 23089. Recuperado de http:// www.nber.org/papers/w23089

Barbaro, M. (2016, octubre 5). Pithy, mean and powerful: How Donald Trump mastered Twitter for 2016. En The New York Times. Recuperado de https://goo.gl/qanp1T

Benoit, W. L. \& Glantz, M. J. (2017). Persuasive attacks on Donald Trump in the 2016 presidential primary. Nueva York: Lexington Books.

Benton, J. (2016, noviembre 9). The forces that drove this election's media failure are likely to get worse. En NiemanLab. Recuperado de https://goo.gl/PLehvw

Berger, A. A. (2017). Marketing and american consumer culture. Nueva York: Springer. 
Bessi, A. \& Ferrara, E. (2016). Social bots distort the 2016 U.S. Presidential election online discussion. First Monday, 21(11), doi: http:// dx.doi.org/10.5210/fm.v21i11.7090

Bovet, A., Morone, F. \& Makse, H. A. (2016, noviembre 7). Predicting election trends with Twitter: Hillary Clinton versus Donald Trump. En arXiv.org. Recuperado de https://goo.gl/t4OroG

Breur, T. (2017). US elections: How could predictions be so wrong? Journal of Marketing Analytics, 4(4), 125-134, doi: https://doi. org/10.1057/s41270-016-0010-2

Bump, P. (2016, octubre 27). Donald Trump's risky plan to use the Internet to suppress Hillary Clinton's turnout. En The Washington Post. Recuperado de https://goo.gl/DptQ6S

Bykowicz, J. (2016, octubre 6). Trump, once a data skeptic, spending millions on data. En AP. Recuperado de https://goo.gl/IGTpVQ

Byrnes, J. (2016, agosto 26). Trump pushes Clinton's 'superpredators' remark. En The Hill. Recuperado de https://goo.gl/VqvUFv

Cellan-Jones, R. (2016, noviembre 11). ¿Fue Facebook clave para el triunfo de Donald Trump? En BBC. Recuperado de https://goo.gl/ TH1hJG

Confessore, N. \& Yourish, K. (2016, marzo 15). \$2 billion worth of free media for Donald Trump. En The New York Times. Recuperado de https://goo.gl/vXgOr4

Conley, B. (2017). Thinking what he says: Market research and the making of Donald Trump's 2016 Presidential campaign. En J. Gillies (ed.), Political marketing in the 2016 U.S. presidential election (pp. 29-48). Nueva York: Springer. 
Coyne, B. (2016, noviembre 7). How \#Election2016 was tweeted so far. En Twitter. Recuperado de https://goo.gl/pze2JN

Dailey, M. (2016, marzo 21). By the numbers: Trump's social media coverage. En SocialFlow. Recuperado de https://goo.gl/kRY0ru

Davis, J. (2017). Presidential campaigns and social networks: How Clinton and Trump used Facebook and Twitter during the 2016 election. Senior Theses and Capstone Projects, 75. Recuperado de http:// scholar.dominican.edu/senior-theses/75

Davis, R., Holtz-Bacha, C. \& Just, M. (eds.) (2016). Twitter and elections around the world. Londres: Routledge.

De la Calle, F. (2016, noviembre 9). Brad Parscale, de campesino de Kansas a gurú digital de Trump. En Vozpópuli. Recuperado de https:// goo.gl/qBAS8Z

Delany, C. (2016, mayo 16). No data for Trump. En epolitics.com. Recuperado de https://goo.gl/a22AsC

Enli, G. (2017). Twitter as arena for the authentic outsider: exploring the social media campaigns of Trump and Clinton in the 2016 US presidential election. European Journal of Communication, 32(1), 5061, doi: https://doi.org/10.1177/0267323116682802

Fraile-Garrido, O. (2016, noviembre 10). Por qué Donald Trump ganó... también en internet. En Socialnautas. Recuperado de https://goo. $\mathrm{gl} / \mathrm{nxvhbw}$

Francia, P. L. (2017). "Going public" in the age of Twitter and mistrust of the media: Donald Trump's 2016 presidential campaign. En J. C. Baumgartner \& T. L. Towner (eds), The internet and the 2016 presidential campaign (pp. 199-218). Lanham: Lexington Books. 
Gallagher, T. (2017). President-elect Trump: Is the past prologue? Society, 54(1), 10-13, doi: https://doi.org/10.1007/s12115-016-0093-6

Goins-Phillips, T. (2016, noviembre 15). Trump digital director: Twitter killed campaign ad buy critical of Clinton. En TheBlaze. Recuperado de https://goo.gl/OyA1Im

González, R. J. (2017). Hacking the citizenry? Personality profiling, big data and the election of Donald Trump. Anthropology Today, 33(3), 9-12, doi: https://dx.doi.org/10.1111/1467-8322.12348

Gottfried, J. \& Shearer, E. (2016, mayo 26). News use across social media platforms 2016. En PewResearchCenter. Recuperado de https:// goo.gl/KM3YNV

Green, J. \& Issenberg, S. (2016, octubre 27). Inside the Trump bunker, with days to go". En Bloomberg Businessweek. Recuperado de https:// goo.gl/9BjK4K

Groshek, J. \& Koc-Michalska, K. (2017). Helping populism win? Social media use, filter bubbles, and support for populist presidential candidates in the 2016 US election campaign. Information, Communication \& Society, 20(9), 1389-1407.

Gross, J. H. \& Johnson, K. T. (2016).Twitter taunts and tirades: Negative campaigning in the age of Trump. Political Science \& Politics, 49(4), 748-754. doi: https://doi.org/10.1017/S1049096516001700

Gutiérrez, C. (2016, julio 6). Clinton vs Trump, la contienda en las redes sociales. En Fortuna. Recuperado de https://goo.gl/vgexW3

Gutiérrez-Rubí, A. (2016, octubre 30). Lo que aprendemos de Trump. En Reforma.com. Recuperado de https://goo.gl/24CxW3

Gutiérrez-Rubí, A. (2016, noviembre 27). Trump en Facebook Live. En Reforma.com. Recuperado de https://goo.gl/OCBoKO 
Haberman, M., Parker, A., Peters, J. W. \& Barbaro, M. (2016, noviembre 6). Inside Donald Trump's last stand: an anxious nominee seeks assurance. En The New York Times. Recuperado de https://goo. gl/OfYeh7

Hall, M. (2016, enero 18). Donald Trump, troll politics and how to win at social media. En The Sydney Morning Herald. Recuperado de https://goo.gl/qTNRV8

Heller, N. (2016, septiembre 27). The first debate of the Twitter election. En The New Yorker. Recuperado de https://goo.gl/nq8Fcw

Hendricks, J. A. (2017). The social media election of 2016. En R. E. Denton (ed.), The 2016 US presidential campaign (pp. 121-150). Nueva York: Springer.

Hermida, A. (2016). Trump and the triumph of affective news when everyone is the media. En D. Lilleker, D. Jackson, E. Thorsen \& A. Veneti (eds.), US election analysis 2016: Media, voters and the campaign (p. 76). Poole: Centre for the Study of Journalism.

Hope, B. (2016, noviembre 9). Inside Donald Trump's data analytics team on election night. En The Wall Street Journal. Recuperado de https://goo.gl/xvz5WB

Johnson, E. (2016, agosto 5). Trump campaign turns to psychographic' data firm used by Cruz. En National Review. Recuperado de https:// goo.gl/us04QN

Kirk, R. \& Martin, S.A. (2017). The dark power of words: stratagems of hate in the 2016 presidential campaign. En R. E. Denton (ed.), The 2016 US presidential campaign (pp. 205-229). Nueva York: Springer.

Kollanyi, B., Howard, P. N. \& Woolley, S. C. (2016, noviembre 17). Bots and automation over Twitter during the U.S. election. En Political Bots. Recuperado de https://goo.gl/5Z5Wwm 
Lambrecht, B. (2016, noviembre 15). Trump's digital ad exec based in San Antonio. En San Antonio Express-News. Recuperado de https:// goo.gl/R28efh

Lang, M. (2016, abril 4). The Donald trumps Clinton, Sanders on social media. En San Francisco Chronicle. Recuperado de https://goo. $\mathrm{gl} / \mathrm{gXbCte}$

Lapowsky, I. (2016, noviembre 19). The man behind Trump's bid to finally take digital seriously. En Wired. Recuperado de https://goo. $\mathrm{gl} / \mathrm{zU} 8 \mathrm{zgp}$

Lee, J. \& Lim, Y-S. (2016). Gendered campaign tuits: The cases of Hillary Clinton and Donald Trump. Public Relations Review, 42(5), 849855, doi: http://dx.doi.org/10.1016/j.pubrev.2016.07.004

Locklear, M. (2016, noviembre 15). Man who led Trump's digital strategy says his SA company earned more than $\$ 90$ million. En News4SA. Recuperado de https://goo.gl/eBLu10

Malec, L., Pavlicek, A. \& Luc, L. (2016). Systematic analysis of social media in 2016 US elections. En Proceedings of the Conference System approaches'16 (pp. 73-79). Praga: Universidad de Praga.

Marshall, J. (2016, agosto 22). The secret behind Trump's comically bad digital campaign? En TPM. Recuperado de https: //goo.gl/SM9ip9

McCabe, D. (2016, junio 27). Trump flexes new digital muscle. En The Hill. Recuperado de https://goo.gl/mLWSSi

Meyer, K. (2016, mayo 8). Trump vs. Clinton vs. Sanders: Who is winning the election on social media? En Business 2 Community. Recuperado de https://goo.gl/JCyqCh

Miller, Z. J. (2016, noviembre 10). Inside Donald Trump's stunning upset. En Time. Recuperado de https://goo.gl/kuqQT8 
Milner, R. M. \& Phillips, W. (2016). Dark Magic: The memes that made Donald Trump's victory. En D. Lilleker, D. Jackson, E. Thorsen \& A. Veneti (eds.), US election analysis 2016: Media, voters and the campaign (pp. 84-85). Poole: Centre for the Study of Journalism.

Mims, C. (2016, octubre 2). How Facebook is dominating the 2016 election. En The Wall Street Journal. Recuperado de https://goo.gl/ qmNQY5

Morin, R. (2016, noviembre 12). Trump says social media was key to victory. En Politico. Recuperado de https://goo.gl/TXZOpn

Morris, D. S. (2017). Twitter versus the traditional media: A survey experiment comparing public perceptions of campaign messages in the 2016 U.S. presidential election. Social Science Computer Review, 35(5), doi: https://doi.org/10.1177/0894439317721441

Ott, B. L. (2017). The age of Twitter: Donald J. Trump and the politics of debasement. Critical Studies in Media Communication, 34(1), 5968, doi: http://dx.doi.org/10.1080/15295036.2016.1266686

Pérez-Martínez, V. M., Rodríguez-González, M. D. \& Tobajas-Gracia, M. (2017). Movilización y participación en Twitter: estudio de caso del hashtag \#SuperTuesday en las primarias presidenciales de EEUU 2016. Revista Latina de Comunicación Social, 72, 679-703, doi: https://doi.org/10.4185/RLCS,72-2017-1186

Persily, N. (2017). Can democracy survive the internet? Journal of Democracy, 28(2), 63-76, doi: https://doi.org/10.1353/jod.2017.0025

Petovel, P. (2016, noviembre 11). La quirúrgica estrategia digital de Trump tiene un responsable: Brad Parscale. En Merca2.0. Recuperado de https://goo.gl/RCkLuk

Plantic, D., Ratkic, H. \& Suput, B. (2017). Comparative analysis of marketing communication strategy on social networks: case study of 
presidential candidates Donald Trump and Hillary Clinton. En Proceedings $20^{\text {th }}$ International Scientific Conference on Economic and Social Development (pp.372-380). Praga.

Rodríguez Andrés, R. (2016). Buscando votos 'online’: las campañas electorales de Hillary Clinton en internet y las redes sociales. En A. E. Kaufmann \& C. Crespo (eds.), Hillary: el poder de la superación (pp. 179-193). Madrid: Catarata.

Rodríguez Andrés, R. \& Ureña Uceda, D. (2011). Diez razones para el uso de Twitter como herramienta en la comunicación política y electoral. Comunicación y Pluralismo, 10, 89-116.

Rosenbaum, S. (2016, octubre 10). Second presidential debate was the most tweeted ever. En New York Post. Recuperado de https://goo. gl/A2krxm

Sáez-Mateu, F. (2017). Democracy, screens, identity, and social networks: The case of Donald Trump's election. American Behavioral Scientist, 61(8), doi: https://doi.org/10.1177/0002764217708585

Silverman, C. (2016, noviembre 16). This analysis shows how fake election news stories outperformed real news on Facebook. En Buzzfeed. Recuperado de https://goo.gl/wKZs8U

Silverman, C. \& Singer-Vine, J. (2016, diciembre 7): Most americans who see fake news believe it. En Buzzfeed. Recuperado de https://goo. $\mathrm{gl} / \mathrm{X} 4 \mathrm{ZuMO}$

Smith, C.A. (2017). Setting the stage: Three dimensions of surfacing for 2016. En J. R. Denton (ed.), The 2016 US presidential campaign (pp. 3-25). Nueva York: Palgrave Macmillan.

Spriester, S. (2016, noviembre 15). President-elect Trump's 'digital guru' calls San Antonio home. En KSAT. Recuperado de https://goo. $\mathrm{gl} /$ snuivB 
Suiter,J. (2016). Post-truth politics. Political Insight, 7(3), 25-27, doi: http:// dx.doi.org/10.1177/2041905816680417

Thomas, M. W. (2015, junio 15). You're hired! Local firm tapped to build Donald Trump for president website. En San Antonio Business Journal. Recuperado de https://goo.gl/Ri6x3e

Tinaquero, J. (2016, marzo 29). La comunicación de Donald Trump: el presidente de las redes sociales. En Medios Sociales. Recuperado de https://goo.gl/FMQZUA

Vogel, K. P. \& Samuelshon, D. (2016, junio 28). Trump's secret data reversal. En Politico. Recuperado de https://goo.gl/vH7oAP

Wagner, K. (2016, noviembre 14). Salesforce CEO Marc Benioff: Without Twitter, you wouldn't have President-elect Trump. En Recode. Recuperado de https://goo.gl/vWA6vw

Wang, Y., Yuncheng, L. \& Jiebo, L. (2016, marzo 9). Deciphering the 2016 U.S. presidential campaign in the Twitter sphere: A comparison of the trumpists and clintonists. En CoRR. Recuperado de https:// goo.gl/94V5eW

Wang, Y., Luo, J., Niemi, R. \& Yuncheng, L. (2016, marzo 27). To follow or not to follow: Analyzing the growth patterns of the trumpists on Twitter. En CoRR. Recuperado de https://goo.gl/wrx2pk

Wang, Y., Luo, J., Niemi, R., Yuncheng, L. \& Tianran, L. (2016, marzo 9). Catching fire via 'likes': Inferring topic preferences of Trump followers on Twitter. En CoRR. Recuperado de https://goo.gl/NR6Jh6

Wells, C., Shah, D.V., Pevehouse, J.C., Yang, J., Pelled, A., Boehm, F., Lukito, J., Ghosh, S. \& Schmidt, J. L. (2016). How Trump drove coverage to the nomination: Hybrid media campaigning. Political Communication, 33(4), 669-676, doi: http://dx.doi.org/10.1080/10 584609.2016 .1224416 
Williams, C. B. (2017). Social media, political marketing and the 2016 U.S. election. Journal of Political Marketing, 16(3), 1-5, doi: http:// dx.doi.org/10.1080/15377857.2017.1345828

Winston, J. (2016, noviembre 18). How the Trump campaign built an identity database and used Facebook ads to win the election. En Medium. Recuperado de https://goo.gl/hizC5k

Zanoni, L. (2016, noviembre 11). El gurú digital de Trump. En El Cronista. Recuperado de https://goo.gl/MOrOHh 